\title{
Atypical Presentation of Multinodular and Vacuolating Neuronal Tumor of the Cerebrum in a Boy
}

\author{
Austin L. Turner ${ }^{1}$ Preston D’Souza' Muhittin Belirgen \\ ${ }^{1}$ School of Medicine, Texas Tech University Health Sciences Center, \\ Lubbock, Texas, United States \\ 2Department of Pediatrics, Texas Tech University Health Sciences \\ Center, Lubbock, Texas, United States
}

Address for correspondence Mohomad M. Al-Rahawan, MD, Department of Pediatrics, Texas Tech University Health Sciences Center, Medical Office Building, 3601 4th Street, Lubbock, TX 79430, United States (e-mail: M.Al@ttuhsc.edu).
The multinodular and vacuolating neuronal tumor (MVNT) was first described by Huse et al in 2013 and recognized by the World Health Organization in 2016.

The pathogenesis of the MVNT is still unclear, with most tumors described as asymmetric focal lesions in the superficial white matter of either the temporal or frontal lobes. Clinical presentations range from asymptomatic lesions to refractory seizures, as well as reported cases of headache, vertigo, paresthesia, and dysarthria. Symptoms have been shown to dissipate following gross or subtotal resection along with a lack of tumor regrowth. ${ }^{1-4}$ Most cases have been reported in the adult population-mean age between 22 and 71 years. ${ }^{1,2,5}$ Currently, immunohistochemical staining patterns, neuroimages, and clinical presentation are necessary for diagnosis.

This case details a 5-year-old boy with a long-standing history of cerebral palsy, spastic gait, and toe walking. His magnetic resonance imaging revealed a stable infiltrative lesion involving the right basal ganglia and posterior limb of right internal capsule, extending inferiorly to the anterior commissure and right hippocampus. T1 hypointensity with $\mathrm{T} 2$ and fluid-attenuated inversion recovery hyperintensity were observed along with a lack of diffusion/perfusion limitations ( - Fig. 1). A slight, asymmetrical decrease in the right cerebral hemisphere was noted. Laboratory studies of blood and cerebrospinal fluid revealed no elevation of $\alpha$ fetoprotein or human chorionic gonadotropin. Hematoxylin and eosin stains of a biopsy specimen displayed many haphazardly arranged neuroepithelial cells with large nuclei and vacuolated cytoplasm ( - Fig. 2A-D). Oligodendrocyte transcription factor 2 was positive in involved glial cells and the vacuolated cells, while Neu-N was negative. Neurofilament stained weakly in few, small vacuolated tumor cells, and leukocyte common antigen was positive in scattered lymphocytes adjacent to vessels. Ki-67 activity was less than $2 \%$ and limited to rare nuclei ( - Fig. 2E, F).
This case encompasses unique features of the MVNT that have not been described in the literature to date. The MVNT was found in a 5-year-old boy, while most are diagnosed in adulthood or postmortem..$^{1-3}$ This brain lesion was found deep in the cerebrum and involved subcortical structures, while those reported in the literature involve the superficial white matter in either the temporal or frontal lobe. ${ }^{1,5}$ The boy's clinical presentation, including his history of cerebral palsy, was not shared among those reported in the literature. While clinical presentation was atypical in this case, imaging and immunohistochemistry were in accordance with the unique patterns specific to the MVNT, allowing for the diagnosis of MVNT to be made.
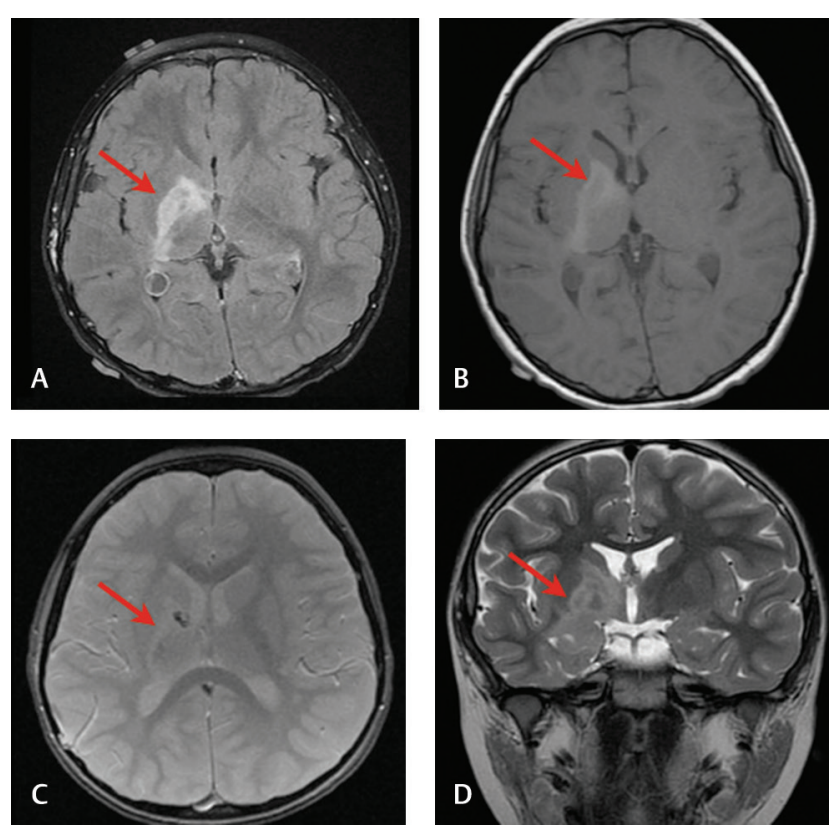

Fig. 1 (A) Preoperative fluid-attenuated inversion recovery, (B) preoperative T1 axial, (C) postoperative multiplanar gradient axial, and (D) preoperative T2 coronal (tumor indicated in red). 

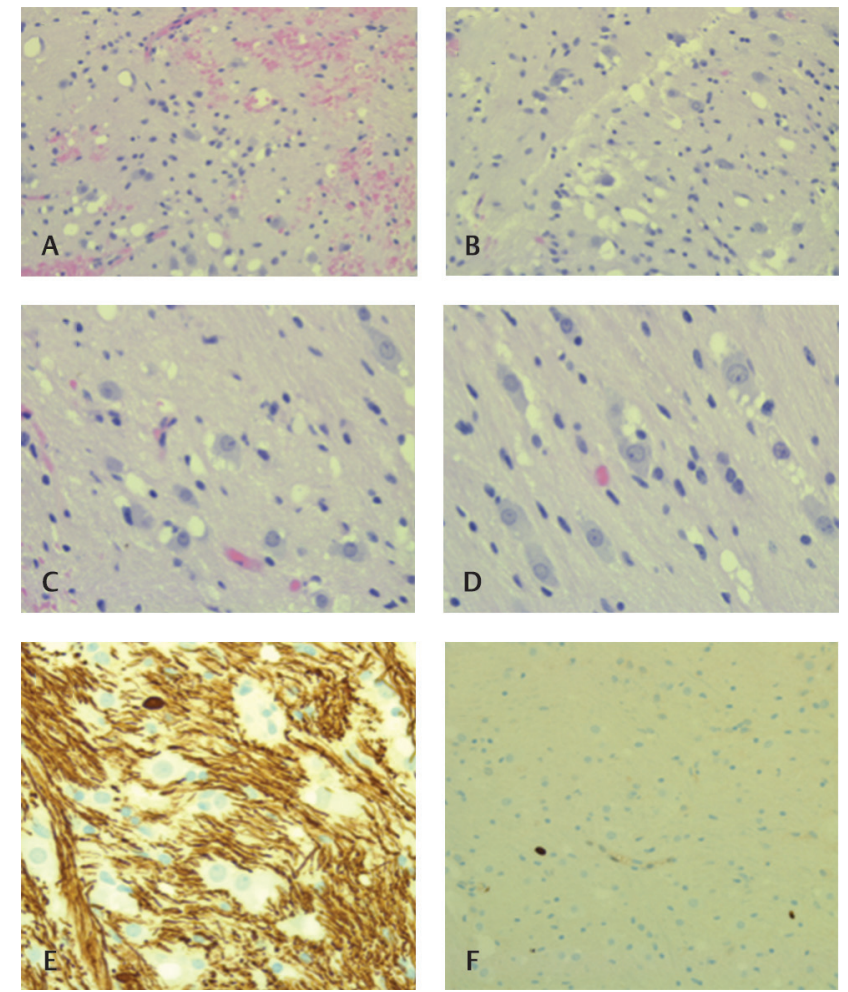

Fig. 2 (A) H\&E stain of tumor cells with vacuolated cytoplasm-low magnification. (B-D) H\&E shows tumor cells with vacuolated cytoplasm-high magnification. (E) Neurofilament IHC shows diffuse positive background with negative vacuolated cell labeling. (F) Ki-67 IHC shows limited positive staining (less than 2\%). H\&E, hematoxylin and eosin; IHC, immunohistochemistry.

\section{Conflict of Interest}

None declared.

\section{References}

1 Alsufayan R, Alcaide-Leon P, de Tilly LN, Mandell DM, Krings T. Natural history of lesions with the MR imaging appearance of multinodular and vacuolating neuronal tumor. Neuroradiology 2017;59(9):873-883

2 Cathcart SJ, Klug JR, Helvey JT. L White M, Gard AP, McComb RD. Multinodular and vacuolating neuronal tumor: a rare seizure associated entity. Am J Surg Pathol 2017;41(7):1005-1010

3 Huse JT, Edgar M, Halliday J, Mikolaenko I, Lavi E, Rosenblum MK. Multinodular and vacuolating neuronal tumors of the cerebrum: 10 cases of a distinctive seizure-associated lesion. Brain Pathol 2013;23(5):515-524

4 Lecler A, Chauvet D, Biassette HA, Savatovsky J. Multiparametric imaging improves confidence in the diagnosis of multinodular and vacuolating neuronal tumor of the cerebrum. AJNR Am J Neuroradiol 2018;39(2):E32-E33

5 Nunes RH, Hsu CC, da Rocha AJ, et al. Multinodular and vacuolating neuronal tumor of the cerebrum: a new "leave me alone" lesion with a characteristic imaging pattern. AJNR Am J Neuroradiol 2017;38(10):1899-1904 